\title{
Strangeness in ultrarelativistic nucleus-nucleus collisions
}

\author{
H. Sorge ${ }^{a}$ \\ a Physics Department, State University of New York at Stony Brook, NY 11794-3800
}

I discuss strangeness production in nucleus-nucleus reactions at ultrarelativistic energies (up to $200 \mathrm{AGeV}$ ). In these reactions matter may be created with densities and temperatures in the transition region between quark-gluon plasma (QGP) and hadron gas. Strange anti-baryon enhancement at $200 \mathrm{AGeV}$ and probably even more so at 10 $\mathrm{AGeV}$ signals importance of interactions beyond hadron gas dynamics. The systematics of strangeness production indicates that energy and baryon density are key variables while the size of the production volume plays no visible role. Analysis of strangeness appears useful to explore thermalization, flow and the post-equilibrium stage in ultrarelativistic nucleus-nucleus collisions.

\section{Introduction}

In this talk I am going to review recent developments to extract information on the properties of dense matter from measurements of strange particles - with emphasis on the phase transition to quark-gluon plasma (QGP). Originally, strangeness enhancement had been suggested as a signature of the quark-gluon plasma (QGP). Most notably, strange anti-baryons have been viewed as particularly well-suited to monitor the formation of a transient QGP in nuclear reactions. Strangeness production in ultrarelativistic nucleusnucleus collisions has been studied experimentally for about one decade now, both at the BNL-AGS and at the CERN-SPS. At the AGS Au beams have been accelerated with an energy of up to nearly $12 \mathrm{AGeV}$. A Pb beam is being utilized at CERN with a beam energy of $158 \mathrm{AGeV}$. Indeed, it has been found that strange hadron yields normalized to pions are enhanced in $A A$ collisions at these energies. A strong strange anti-baryon enhancement has been experimentally observed for central $\mathrm{S}$ collisions on $\mathrm{S}$ and heavier targets at $200 \mathrm{AGeV}$ [2:3]. An even stronger enhancement of $\bar{\Lambda} / \bar{p}$ in $\mathrm{Si}+\mathrm{Au}$ and $\mathrm{Au}+\mathrm{Au}$ reactions at $10-15 \mathrm{AGeV}$ is suggested by some preliminary data. Two sets of $\bar{p}$ data measured by detectors with different sensitivity to feed-down from weak (hyperon) decays can be reconciled with each other only if the $\bar{\Lambda} / \bar{p}$ ratio is extremely large, on the order of 5-6 [4]. Over the years it has become clear from the systematics of measurements and from theoretical considerations that strangeness enhancement is not as clearly linked to a QGP as initially thought. The theoretical debate about production mechanisms will probably go on for a while, because calculations are messy due to the intrinsically nonperturbative nature of hadronization. Therefore I will discuss later whether one can distinguish production mechanisms experimentally.

Recently, it has been argued that central nucleus-nucleus collisions at high energies 
produce a hadronic state in chemical equilibrium at freeze-out [5]. This would be, of course, a radical 'solution' to the debate about production mechanisms. An equilibrium state does not keep a memory of how it has been produced. The information about its creation would be lost. The situation is not so hopeless as it looks like. By lowering the beam energy or the projectile-target masses one can impose more unfavorable conditions for chemical equilibration. The final-state dynamics in nuclear reactions adds another facet to the thermalization issue. 'Freeze-out' in heavy-ion collisions is not a state but a stage. It is sometimes argued that only if the final hadronic stage can be characterized in terms of thermal and hadrochemical equilibrium one can hope to describe the earlier stages in terms of equilibrium concepts (see e.g. [6]). However, already several years ago Bebie et al. have demonstrated in a thorough study of the decoupling conditions in ultrarelativistic $A A$ collisions that the various equilibria break-down necessarily in the final stages [7]. For instance, strange hadron yields go out of equilibrium first, because strangeness production and absorption rates are getting small in comparison to the (quasi-elastic) collision rates. Hydrodynamic and transport calculations point to the existence of a 'post-equilibrium' stage in ultrarelativistic $A A$ reactions. One can utilize the effect that different hadron species decouple sequentially to study the time evolution of the system. For instance, this helps to address the question whether the observed collective flow develops 'early' or 'late'. Such information is critical for studies of the equation of state in the phase transition region 8$]$.

\section{Prehadronic stage: strings, partons or QGP?}

Hadronic observables can probe only indirectly the nature of the prehadronic stage. Hadronization and further interactions in the hadronic phase will leave their imprints on chemical composition and phase space distributions. With this caveat strange antibaryons are probably the most useful species to gain information on the prehadronic stages of nuclear collisions. Interactions in a hadron gas are too slow to result in a sizable creation rate. This was conjectured first based on calculations for a thermal system [1]. Later, it was confirmed by transport calculations as well. The hadronic interactions in the pre-equilibrium stage are very energetic according to calculations with a transport model like RQMD. Initial interactions between mesons and in-going baryons are characterized by effective temperatures of more than $300 \mathrm{MeV}$ at CERN energy [9]. Employing $\Delta E \sim 3 T$ tells that $\bar{s} s$ creation at this stage is not severely suppressed in channels like $\Lambda K$. However, even these hard initial hadronic collisions do usually not provide the necessary energy of 2-4 GeV needed to create a strange baryon pair.

Since energy is the key variable it is natural to expect that the earlier and denser prehadronic stage provides more favorable conditions for anti-baryon production. The models which have been successfully tested in $h h, h A$ and $A A$ collisions at the ultrahigh beam energies $\gg 10 \mathrm{AGeV}$ (RQMD , VENUS, QGSM and other versions of DPM models) employ some sort of multiple string excitation mechanism for the prehadronic (quark matter) stage. The strings represent the coherent color-electric field which is radiated from the in-going color charges. The soft modes of these fields are squeezed by the surrounding non-perturbative vacuum into flux-tubes essentially along the beam axis.

If the system is heated quickly - e.g. by coupling to hard partonic modes - string-like 
configurations may 'melt' and give way to a QGP. Whether this happens at presently explored beam energies is still an open question.

Beyond some 'critical' string density independent fragmentation is expected to break down. In RQMD [10] (and also in the Spanish version of the DPM [11) strings will fuse into color 'ropes' if they are overlapping. One consequence of the strong coherent gluon fields in the rope is their fast screening by quark pair production. The strong $s \bar{s}$ enhancement in the coherent rope fields is displayed in Fig. 1. $(p, q)$ characterize the $S U_{3}$ color charge creating the rope gluon field. $p$, respectively $q$ can be thought of as the unscreened quark (anti-quark) sources of the field. Unlike in a string, the end of ropes contain usually many diquarks and anti-diquarks with the same color charge as (anti-)quarks. This is

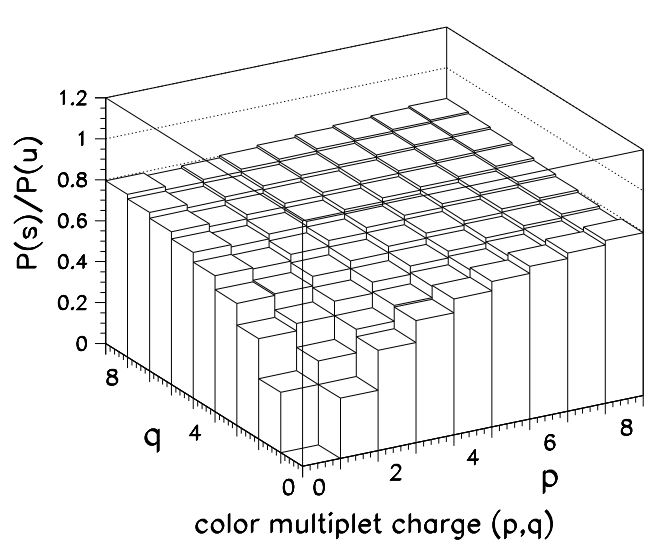

Figure 1: Rope decay:

strangeness suppression the consequence of the $S U_{3}$ group combinatorics, e.g. $3+3=6+\overline{3}$. The most drastic change of rope compared to independent string fragmentation is therefore in the strange anti-baryon sector, because strangeness and (anti-)diquark enhancement both contribute here. While independent string fragmentation fails completely in comparison to the data for $\mathrm{S}$ induced reactions at $200 \mathrm{AGeV}$ the results with color rope (or string fusion) included agree rather well with measurements [9]. First preliminary results from NA49 [12] and WA97 (see K. Safarik's contribution to the Proceedings) for central Pb(158AGeV) on Pb reactions are consistent with the RQMD predictions of anti-baryon densities published in [13. Anti-baryon rapidity distributions $\left(\bar{p}, \bar{\Lambda}, \bar{\Xi}^{0,+}\right)$ in central $\mathrm{Pb}(160 \mathrm{AGeV})+\mathrm{Pb}$ collisions which have been calculated using RQMD in three different modes are displayed in Fig. 2. The three different operation modes of RQMD have been ropes and rescattering switched off ('NN mode', dashed line), rope fragmentation included (dotted line) and ropes and hadronic rescattering both included which is the default mode (straight line). The $\Xi$ yield as calculated with the default version 2.1 of RQMD increases by a factor of 7.7 compared to the result in the 'NN mode'. The increase would be even a factor of 13.3 if anti-baryons would not be absorbed in the later hadronic stage.

Capella has taken a somewhat different path to explain strangeness and diquark enhancement from string fragmentation in $A A$ collisions 14. Elementary $h h$ collisions produce mostly strings with the in-going valence quarks at their ends. However, in $A A$ collisions additional strings are formed with sea quarks at the ends. With an assumption about a rather large strangeness fraction $s / u=0.5$ and some $B-\bar{B}$ content in the nucleon sea he can explain measurements for $\mathrm{S}+\mathrm{S}$ and $\mathrm{S}+\mathrm{W}$ collisions at $200 \mathrm{AGeV}$. It is an interesting question whether the large diquark and strangeness content in this model mimicks the effect of rope formation or the other way around.

A completely statistical (microcanonical) approach to the fragmentation of large density spots which emerge in a percolation of strings has been developed in VENUS [15]. The chemical composition depends on the choice for the energy at which the 'droplet' is 
converted into hadrons. VENUS iterates this scheme to simulate hadronic interactions. Since only final hadron yields from VENUS have been published it is impossible to directly compare the chemical compositions from initial droplet decay and rope fragmentation. Qualitatively, trends - strangeness and even more pronounced anti-baryon enhancement - are similar in both approaches. Note that in RQMD anti-baryon annihilation on baryons is based on the free $\bar{p} p$ cross sections and in VENUS on a smaller - energy-independent and universal - interaction strength.

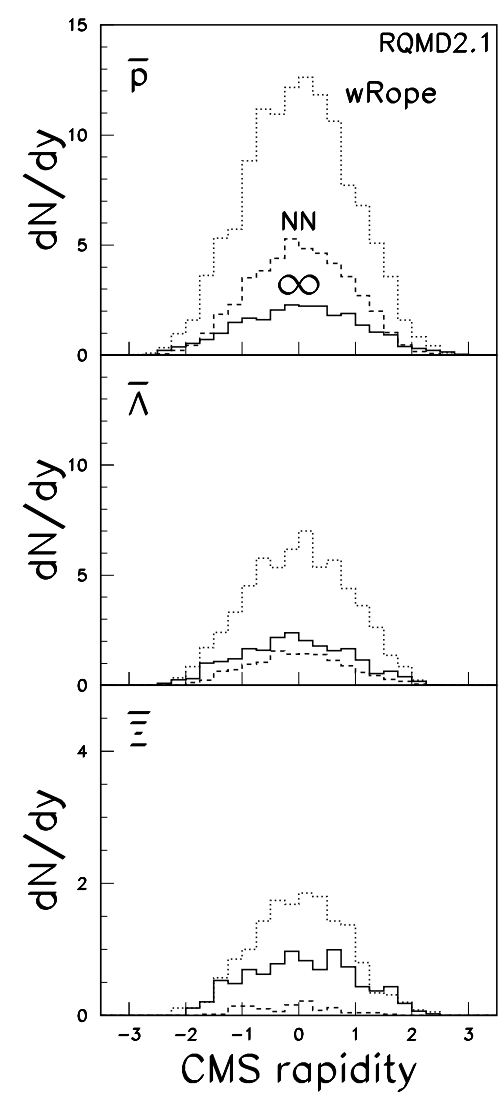

Figure 2: $\mathrm{Pb}(160 \mathrm{AGeV})+\mathrm{Pb}$, Anti-baryons from RQMD
Very recently, it has been claimed that one can explain soft particle production without invoking strings - by straightforward application of perturbative QCD in a parton cascade model $(\mathrm{PCM})$ [16]. However, a 'string effect' is effectively put into this model using arbitrary cutoff prescriptions for the infrared-divergent parton cross sections. The cut-offs have been finetuned to generate the rapidity densities which have been experimentally observed in $h h$ collisions. The place in which hard parton-parton scatterings and not the cut-off's determine the model's results are hadrons with large transverse momenta. Incidentally, $\mathrm{PCM}$ generates too many pions with $p_{t}>3 \mathrm{GeV}$ - a factor 5 compared to experimental WA98 data. The failure points either to an overestimation of multiple hard collision effects in PCM or an underestimation of the energy loss which a fast parton experiences in dense matter due to lack of soft interactions. The parton cascade combined with a hadronic 'after-burner' (HIJET) agrees well with preliminary data from NA49 for $K_{S} / \pi$. Interestingly, the strangeness enhancement (compared to $p p$ ) seems to have been produced mainly in the hadronic stage. A similar trend has been found in RQMD comparing strangeness enhancement in rope fragmentation with the contribution from hadronic rescattering [13]. Concerning strange antibaryon production the parton cascade model shows a weaker enhancement for central $\mathrm{Pb}(158 \mathrm{AGeV})$ on $\mathrm{Pb}$ reactions than VENUS (and RQMD). This can be seen from the model comparisons of $\bar{\Lambda} / \bar{p}$ in [1] .

Some statistical approaches have been developed in recent years to describe the hadronization of a quark-gluon plasma microscopically, mostly in terms of quark coalescence (see e.g. [17], [18]). These coalescence models predict an $\bar{\Omega} / \Omega$ ratio $\geq 1$. The result is generic for this class of models, because there are as many $s$ as $\bar{s}$ quarks, independent on the degree of strangeness equilibration. Furthermore, three $s$ quarks fuse with same probability as three $\bar{s}$ quarks into an $\Omega$, respectively $\bar{\Omega}$ state. So far, no further interactions between 
hadrons are included in these models.

The differences in the treatment of anti-baryon annihilation in the various quoted approaches are clearly larger than for the production mechanisms. It ranges from no annihilation at all to full strength as determined from free $\bar{p} p$ cross sections. Therefore it is useful to explore the strength of anti-baryon absorption experimentally. For instance, measurement of 'anti-flow' in non-central collisions can clarify how strongly various antibaryon species are absorbed by the co-moving baryons [19. Another idea is to study anti-baryon-proton correlations in the final state. Note that anti-baryon studies in the 'stopping region' (up to $30 \mathrm{GeV}$ ) are particularly useful in this respect. Only a small fraction of anti-baryons survives in the extremely baryon-rich medium produced at these energies. Comparison between models and experimental data has lead to quite detailed insights. Most interestingly, the the $\bar{p} p$ annihilation cross section which rises strongly at low energy may be somewhat screened in dense matter [20]. In most recent RQMD versions this effect is attributed to $\bar{B}-B$ 'molecule' states close to threshold which have a finite life time before they radiate into mesons. In the medium they may break up again if they scatter with other hadrons.

\section{Equilibration: is $\mathrm{p}+\mathrm{A}$ more interesting than $\mathrm{Pb}+\mathrm{Pb}$ ?}

As pointed out already as a caveat interactions in the hadronic phase may strongly alter the primordial anti-baryon distributions. This holds certainly true for anti-baryons in baryon-rich matter, because their annihilation rate based on free-space cross sections is huge. We know from experimental data that a sizable fraction of the in-going baryons is stopped. Therefore annihilation reactions may possibly wash out the interesting information from the earlier stage. A very strong effect of anti-baryon absorption in central $\mathrm{Pb}(160 \mathrm{AGeV})$ on $\mathrm{Pb}$ collisions can be inferred from the RQMD results in Fig. 2. Note, in particular, that the final $\bar{p}$ is even below the result from the $N N$ mode of RQMD. All strong enhancement due to initial rope formation is gone! This provides a useful lesson about the study of production mechanisms. Heavy projectile-target combinations with beam energy as large as $160 \mathrm{AGeV}$ are probably unsuitable for this purpose, because strong chemical equilibration destroys the memory of the earlier stages.

If we want to shed more light on the mechanisms which are responsible for the observed strange anti-baryon enhancement we have to go to the smaller systems. An analysis of chemical equilibration in the strange (anti-)baryon sector for $\mathrm{S}(200 \mathrm{AGeV})$ on $\mathrm{W}$ collisions has been made in 21]. The main result can be extracted from Fig. 3 in which the total and the net contributions to $\Xi$ and $\Xi$ are displayed. The upper part shows the important net contributions of the different production mechanisms $(M B=$ meson-baryon collisions and so on). The lower part breaks the $M B$ contributions up into two parts, from intermediate states with cascade $\left(\Xi^{\star}\right)$, respectively hyperon $\left(Y^{\star}\right)$ quantum numbers. It is clear that reactions with these intermediate quantum numbers are responsible to equilibrate hyperon and cascade states, e.g. $\Lambda \bar{K} \longleftrightarrow \Xi \pi$ (and analogously for the anti-particles). Even in this light ion induced reaction the back and forth between the different baryon states seems to be rather strong. It is apparent that the total contributions in the central rapidity region are considerably larger than the net contributions. This means in turn that these strange baryon species must be locally rather close to chemical equilibrium values. 


\section{(Anti-) E sources in $\mathrm{S}+\mathrm{W}$}
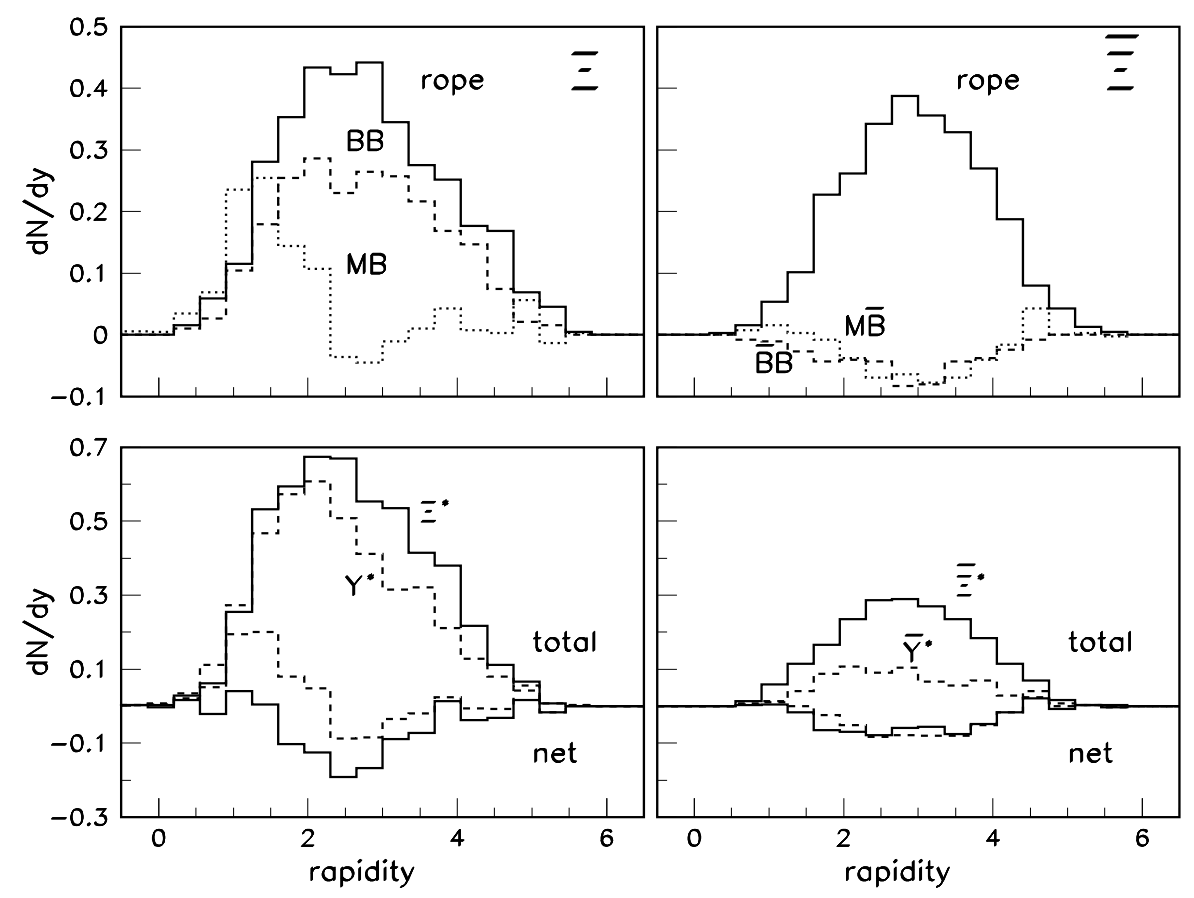

Figure 3. Sources of $\Xi$ and $\Xi$ in $\mathrm{S}(200 \mathrm{AGeV})$ on W from RQMD [21]

The microscopic calculations with RQMD provide some justification for the equilibrium assumptions underlying thermal fireball fits. An example of these fits is taken from Cleyman's paper [22] and shown in Fig. ․ One of the truely intriguing aspects is that the temperature extracted from the fit of strange (anti-)baryons is very large, close to 190 $\mathrm{MeV}$. This tells immediately that $T$ cannot be a real freeze-out temperature of a hadronic gas. A hadron density of approximately $1 \mathrm{fm}^{-3}$ is so high that particles cannot decouple. A more reasonable conclusion is that the yields of these species do not change appreciably any more below this temperature (chemical freeze-out). According to the transport calculation with RQMD and other models [22] early chemical freeze-out is caused by the small hadronic strangeness creation and absorption rates. The relevant cross sections are typically just a couple of millibarns. The range of extracted chemical freeze-out temperatures is very close to - maybe even above - the phase transition temperature to the QGP. However, no QGP phase transition is incorporated into RQMD. The good agreement of RQMD with strangeness measurements could be an indication that the properties of hadronic matter change very smoothly into QGP properties in the transition region.

Sometimes - as in Fig. A - an extra parameter is introduced to account for suppression of hadrons containing strange valence quarks compared to equilibrium values. This strangeness suppression parameter is 0.7 in Cleyman's fit, roughly a factor of 2 larger than in corresponding fits to particle yields from $p p$ or $e^{+} e^{-}$interactions. It seems to indicate that the systems created in $S$ induced reactions are much closer to chemical equilibrium than the small systems. A very important question in this respect is whether there is some threshold effect for strangeness enhancement as a function of system size. Clearly, 
strangeness production depends on the energy density achieved. Does it also depend on whether the size of the production volume exceeds the confinement scale of one fermi? Does it depend on the degree of thermalization which is achieved in these reactions? These questions are of uttermost importance in the context of strangeness as a signature for QGP in the thermodynamic sense. The place to look for answers to these questions is $p A$ collisions, because the fire cylinder in these reactions has a small transverse size given roughly by the radius of the in-going projectile proton. Furthermore, the life time of the system created in $p+A$ collisions is expected to be rather small which works against equilibration.

Recently, it has been fiercely debated whether $p$ collisions with nuclei as small as $S$ show 'strangeness enhancement' or not [24,25]. The answer depends somewhat on the definition. Integrated $K / \pi$ seems not much affected by the presence of nuclei. Some $K^{+}$enhancement is seen at backward rapidities which is expected from rescattering effects leading to associated production [26]. This is consistent with the observation that hyperons scale non-linearly with mass number. The most remarkable $p A$ result concerning strangeness has been published only recently. The multiple strange antibaryon yields reveal that production volume and degree of thermalization are not relevant variables for their production. WA94 finds

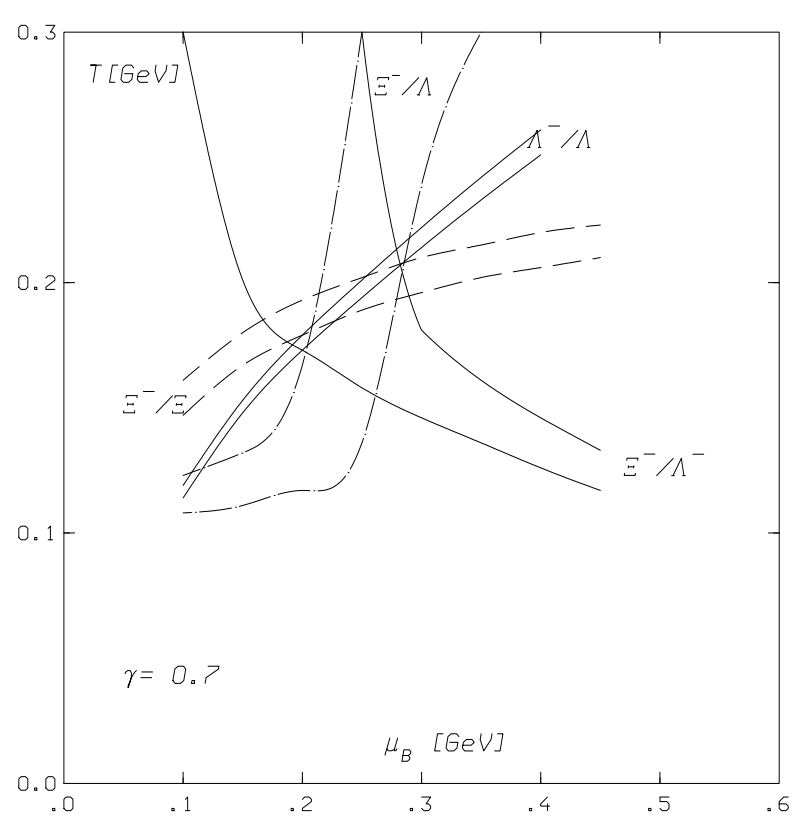

Figure 4: Fireball fit to $\mathrm{S}(200 \mathrm{AGeV})$ on W [22] that the $\bar{\Xi} / \bar{\Lambda}$ value already in $\mathrm{p}(200 \mathrm{GeV}$ ) on $\mathrm{S}$ (and W) is intermediate between the $p p$ and the $S+S / W$ results [27. This means that two overlapping strings as typically produced in $p+S$ collisions are sufficient to create a strong departure from particle ratios in $p p$ collisions. It was already discussed in [26] that ropes affect strongly the strange anti-baryon yields in $p+A$ reactions. String fusion to color ropes provides an example in which strangeness creation is sensitive to the local energy densities but not to the production volume. Whatever the best effective theory to describe $p A$ and $A A$ reactions one conclusion can be safely drawn from the experimental data alone. Strange hadron yields per pion change smoothly from $p p$ to $p A$ and $A A$. No clear threshold behaviour is observed. This is bad news and good news at the same time. The question whether a quark-gluon plasma state of macroscopic dimensions in space and time is created in $A A$ collisions can probably not be answered by looking at the strange hadrons. On the other side, all information on strange hadrons produced in the small systems point to strong influence of variations in energy density on the particle ratios. The observed leveling-off of strange particle ratio like $\Xi / \bar{\Lambda}$ in data taken with the $S$ and the $P b$ beam [28] tells that the truely heavy-ion collisions do not probe so much the production mechanisms any more but the subsequent equilibration stages. An experimental program with $p$ and 
very light ion beams at the CERN-SPS energies is required if one would like to study strangeness production mechanisms carefully. This is also warranted in view of present experimental uncertainties and conflicting data sets, even on the level of $p p$ collisions. Another direction to proceed would be to study heavy-ion collisions at lower beam energies. Most interestingly, $\bar{\Lambda} / \bar{p}$ seems to be much larger at the beam energy of $10 \mathrm{AGeV}$ than at $200 \mathrm{AGeV}$. What about the ratio $\bar{\Xi} / \bar{\Lambda}$ in $\mathrm{Au}$ on $\mathrm{Au}$ at $10 \mathrm{AGeV}$ ? Nobody knows the answer. There must be a lower beam energy at which the inversion that heavier antibaryons are populated more frequently than the lower ones goes away due to lack of phase space. Enhancement (and absorption) effects are expected to be much more pronounced at lower beam energies which should help to clarify their dynamical origins.

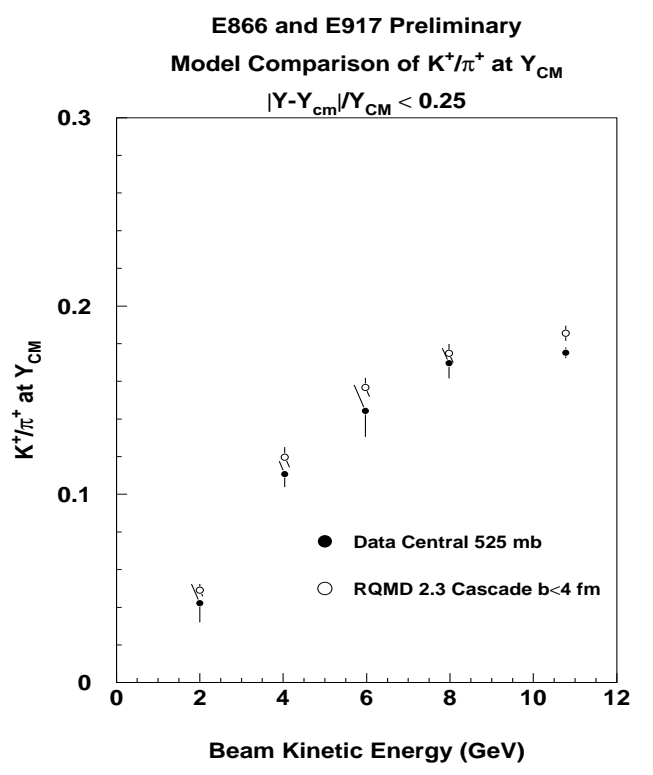

Figure 5: $\quad K / \pi$ in $\mathrm{Au}+\mathrm{Au}, 2-12 \mathrm{AGeV}[29]$

So far I have focused on equlibration in the strange (anti-)baryon sector. Of course, the kaon degrees of freedom should get equilibrated much more easily because of the comparably smaller masses. Arguments have even been put forward that the $K / \pi$ ratios measured in $A A$ collisions at CERN energies signal the QGP formation. Some authors argue that the $K / \pi$ ratio is anomalously large [25] which is based on a comparison to $p p$ data. On the other side, it is also sometimes suggested (even by the same authors) that the ratio is anomalously small [30]. This is based on the argument that the hadronic fireball fits work well in the strange (anti-)baryon sector but predict a too large $K / \pi$ ratio [31] in $S$ induced collisions. Indeed, the observation of a 'pion excess' indicates an interesting non-equilibrium effect. A simple reason has been extracted from the RQMD transport calculations as was pointed out in [21]. The source size of pions is systematically larger than the source of kaons and (anti-)baryons. On the one side, this is a consequence of the different production mechanisms. For instance, anti-baryons are produced more centrally in the impact parameter plane than pions, because their formation rate is more sensitive to the higher energy densities in the interior (the overlapping strings and formed ropes in the RQMD model). Furthermore, the pion 'fluid' expands considerably faster than the heavy hadron fluid [32]. A comparison of RQMD predictions with recent preliminary NA49 data for central $\mathrm{Pb}(158 \mathrm{AGeV})$ on $\mathrm{Pb}$ collisions [8] establishes that this type of non-ideal hydrodynamic expansion is essential for the good agreement between the shape of the calculated transverse mass spectra and data. Note also that RQMD has predicted not just the shape but also the measured $K_{S} / \pi$ ratio correctly.

A test of the idea whether the $K / \pi$ ratio can be linked to the QGP formation is to lower the beam energy and look for a discontinuity in the excitation function. Such a test has been realized recently at the BNL-AGS by varying the beam energy between 2 and $12 \mathrm{AGeV}$ (see C. Ogilvie's talk). The data are still very preliminary and are shown in 
Fig. 5. They are compared in this Fig. with the predictions from the RQMD model. The agreement is very good. Neither data nor model show any discontinuity in this variable.

One can go back to the model and look for the dominating source of strangeness enhancement [33]. It turns out that the crucial mechanism is the hard collison spectrum in the pre-equilibrium stage leading to the formation of excitations (resonances or strings), predominantly via meson-baryon interactions. Since the resonances are progated themselves even larger mass excitations may be created in multi-step processes.

These processes, e.g.

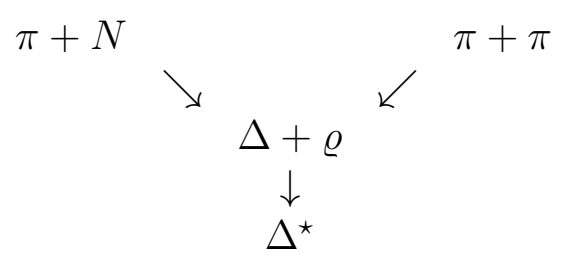

are of huge importance for strangeness creation, because intermediate resonances act as an 'energy storage'. Such multi-body hadronic interactions are only frequent in a system of sufficient density of roughly $1 \mathrm{fm}^{-3}$, because the life time of the intermediate states amounts to typically 1-2 fm/c only.

Resonances, in particular the $\Delta(1232)$, impact the dynamics in $A A$ collisions already at beam energies of 1-2 AGeV 34. Going to higher beam energies means at first that more and more resonance states can be excited. This is reflected in the various attempts for a theoretical description of $A A$ collisions in the region of beam energies above $1 \mathrm{AGeV}$. A transport model like RQMD contains a whole tower of resonance excitations, basically all confirmed resonance states up to a mass of $2 \mathrm{GeV}$ (including the missing members of $S U(3)_{F}$ multiplets required by the quark model). Resonance matter formation seems to be a crucial aspect of the physics of hot, dense hadronic matter. The resonance spectrum is related primarily to the confinement property of QCD. In equilibrium, the excitation of the resonance states with their large degeneracy factors leads to a strong increase of energy (and entropy) density within a small temperature range 150-170 MeV. The most extreme variant of such an approach is the Hagedorn gas with an exponentially increasing spectrum of states as a function of mass which leads to a limiting temperature. Is resonance matter just a way of describing the 'mixed phase' in the transition region employing hadronic degrees of freedom? In fact, excitation of resonances provides even a path to chiral symmetry restoration. Glozman and Riska argue that the splitting between parity doublets which characterize the amount of spontaneous breaking of chiral symmetry goes to zero for higher lying flavor multiplets [35]. It is an open and interesting question how far one can push the idea of duality between descriptions on the hadronic and the quarkgluon level in the transition region. Another interesting idea is that hadron properties e.g. masses and decay widths - themselves might change in the dense medium. Since these properties are tied to the spontaneous breaking of chiral symmetry, partial restoration of this symmetry at finite density and temperature may lead to these changes. Furthermore, effective theories like chiral perturbation theory predict medium effects - even without chiral restoration. Studies of strangeness might help to shed some light on this question. Does subthreshold production of $K^{-}$in $A A$ collisions at GSI-SIS energy give an indication for a dropping $K^{-}$mass? This is an exciting possibility which certainly deserves thorough 
experimental and theoretical studies (see G.Q. Li's talk, [36]).

\section{Strangeness and the post-equilibrium stage}

The post-equilibrium stage develops in $A A$ collisions when the reaction rates are not large enough to keep the expanding system in equilibrium. (Of course, it is possible that local equilibrium has not been achieved at all during intermediate stages.) The criterion from kinetic theory is that the associated relaxation time becomes larger than the expansion time scale. In particular, in a relatively baryon-number poor environment as created at CERN energies this is going to happen quickly for the rare anti-baryons. Their absolute number probably freezes-out rather early. Lateron the strangeness content goes out of equilibrium, because it changes too slowly. The effective pion number (counting rho as two pions and so on) is freezing-out approximately at the same time as strangeness. The reason is that reactions of the type $\pi \pi \pi \pi \leftrightarrow \pi \pi$ are characterized by small cross sections, too. Furthermore, pion number may change only by at least two units in elementary reactions in the meson sector (from $G$ parity). Kinetic equilibrium in the expanding hadron gas can probably be maintained for a longer time. No kinematic threshold prevents the occurence of elastic reactions. Since hadrons with strange valence quark content have typically smaller elastic or quasi-elastic cross sections, their interaction tends to cease earlier. Another simple rule based on quark counting and empirical observation is that mesons have usually smaller interaction rates than baryons. Of course, the Goldstone theorems makes sure that pion interactions at low energies are dynamically suppressed. Therefore pions will decouple earlier than for instance omega mesons or nucleons. Transport calculations have been analyzed to study the post-equilibrium effects very thoroughly [23, 32. The spectrum of freeze-out times for different hadron species produced at midrapidity in central $\mathrm{Pb}(160 \mathrm{AGeV})$ on $\mathrm{Pb}$ collisions according to RQMD calculations is displayed in Fig. 6. It confirms the stated trends. Note that the double-strange $\Phi$ mesons decouple from the system about $12 \mathrm{fm} / \mathrm{c}$ earlier than the nucleons.

An impressing body of evidence has been accumulated in recent years that models like RQMD, VENUS and ARC produce a final source which is a very good approximation of the real source in phase (position and momentum) space. In this respect HBT analysis (see W. Zaic's talk) and nuclear cluster calculations were very important, because the spacetime characteristics of the sources as calculated in these models is completely determined by the interactions. The realistic simulation of the final dilute stages in nucleus-nucleus reactions - including the break-down of equilibria - appears to be an important reason why the microscopic models are so successful compared to experimental data. This is most clearly revealed by comparison to hydrodynamic calculations which usually assume the existence of local equilibrium until the final freeze-out and therefore lack the sequential break-down of equilibria. For instance, momentum distributions calculated with a hydrodynamical code for $S$ induced reactions at $200 \mathrm{AGeV}$ are presented and discussed in [37]. It is apparent from the comparison to experimental data that sequential freeze-out could considerably improve the agreement. In this particular calculation a 'compromise' value for the freeze-out temperature has been chosen. This means that strange anti-baryons like $\bar{\Lambda}$ are underpredicted, because the chosen temperature is already too 'cool'. On the other side, the hadron spectra are generally too soft compared to the measurements indicating 

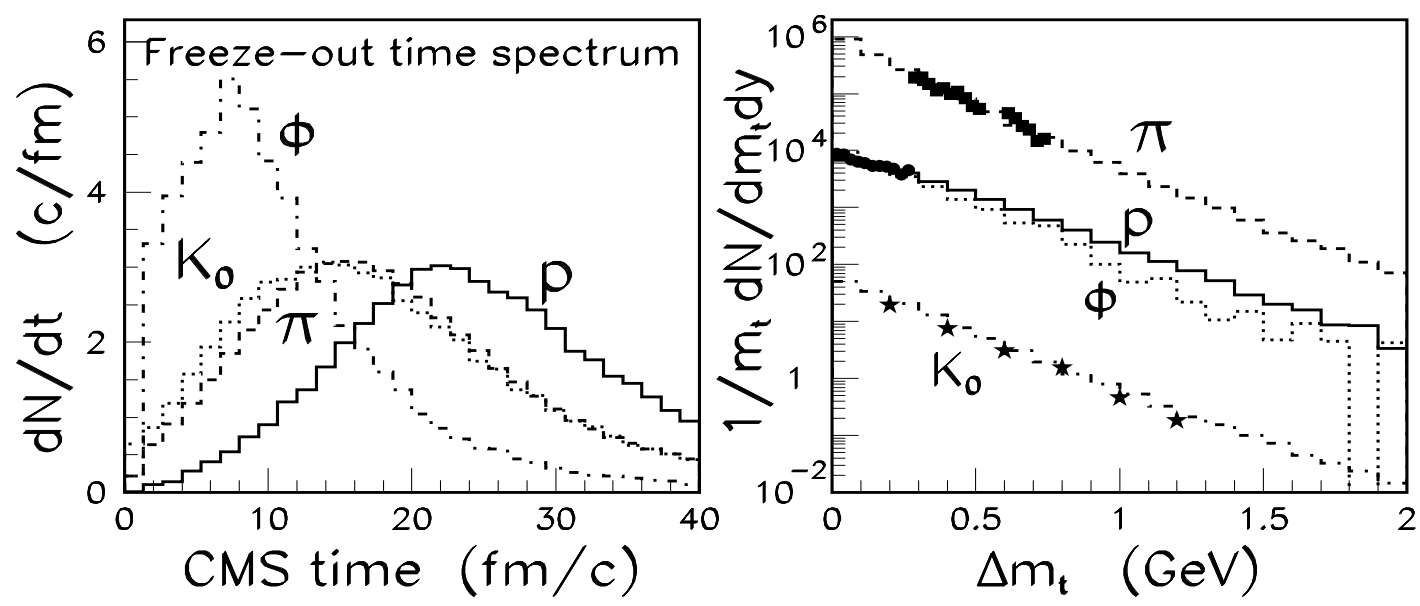

Figure 6. Freeze-out time and transverse mass $\left(m_{t}\right)$ spectra of hadrons at midrapidity calculated with RQMD 2.1. $m_{t}$ spectra are compared to preliminary NA49 data. Yields have been renormalized for display reason.

that the system freezes-out too early: the transverse flow has not yet fully developed. Presently, several groups are studying how the freeze-out stage can be better simulated either intgrated into or as an 'after-burner' to the hydrodynamics calculations.

The importance of the post-equilibrium stage increases strongly with the masses of projectile and target. As pointed out in [13] the source which is produced in $\mathrm{Pb}+\mathrm{Pb}$ reactions lives much longer in the hadronic stage than the source created e.g. in $\mathrm{S}+\mathrm{Pb}$. Three-dimensional expansion becomes effective only much later in the heavier system. The reason is that the initial transverse size of the reactione zone is larger by almost a factor of four. (In the limit of infinite transverse size expansion would be purely longitudinal.) Furthermore, the larger system cools down to lower kinetic temperatures [21], because the initial size affects also the decoupling conditions.

Let me give just one example how the sequential decoupling of hadron species from the medium can be utilized to gain information on properties of the medium. Flow analysis is one of the most promising areas to study the physics of the phase transition. Expansion becomes very 'soft' in the transition region affecting the flow of the matter. There are many speculations whether the observed rather hard transverse momentum spectra in $\mathrm{Pb}(160 \mathrm{AGeV})+\mathrm{Pb}$ reactions - slope parameters close to $290 \mathrm{MeV}$ for (anti)baryons - reflect an 'early' (partonic?) flow or 'random kick' 38 or - alternatively - develop only very late in the hadronic stage. A comparison of $\Phi$ and $p$ spectra is very useful, because these two species have practically the same mass. The random $p_{t}$ component which they acquire is therefore the same in a thermal (or hydrodynamical) framework. RQMD predicts that the $\Phi$ spectrum is in fact considerably softer (slope 225 $\mathrm{MeV}$ ) than the $p$ spectrum with slope $280 \mathrm{MeV}$ (see the rhs of Fig. 6). This seems to indicate that the additional flow seen in $\mathrm{Pb}+\mathrm{Pb}$ (beyond what has been observed in $\mathrm{p}+\mathrm{A}$ and $\mathrm{S}+\mathrm{A}$ at $200 \mathrm{AGeV}$ ) is produced rather late only, after the $\Phi$ has already decoupled. The results support the idea that initially the source at CERN energies expands very 
softly into transverse directions. It is another (interesting) question whether this is a preequilibrium effect (e.g. from ropes) or manifestation of the 'softest point' in the Equation of State [39]. A general lesson to be drawn from recent experience gained with $A A$ reactions at the ultra-high but also at the relativistic $(1 \mathrm{AGeV})$ energies [40] is that strange hadrons are a very valuable tool to disentangle the various contributions to the forces which drive the collective flow.

\section{Conclusions}

I find evidence from the systematics of measurements and theoretical studies that strangeness enhancement per se is not a signature of QGP formation in ultrarelativistic nucleus-nucleus collisions. On the other side, strange anti-baryon enhancement at $160 \mathrm{AGeV}$ - and probably even more so at $10 \mathrm{AGeV}$ - signals importance of interactions beyond (quasi-free) hadron gas dynamics. If one wants to study 'threshold behaviour' carefully one has to go back to small systems like $p+A$ or to lower beam energies. Concerning the heavy-ion reactions emphasis has shifted recently from study of production mechanisms to equilibrium and post-equilibrium physics. This reflects that strange hadrons - even the multiple strange anti-baryons - are produced frequently enough to achieve a large degree of chemical equilibration in the center of the 'fireball'.

This work has been supported by DOE grant No. DE-FG02-88ER40388.

\section{REFERENCES}

1. P. Koch, J. Rafelski, and B. Müller: Phys. Rep. 142 (1986) 167.

2. J. Bartke et al. (NA35 Collab.): Z. f. Phys. C48 (1990) 191; J. Bächler et al. (NA35 Collab.): Z. f. Phys. C61 (1994) 551.

3. S. Abatzis et al. (WA85 Collab.): Phys. Lett. B244 (1990) 130; Phys. Lett. B259 (1990) 508; Phys. Lett. B270 (1991) 123.

4. J.G. Lajoie (for the E864 Collab.): Proceedings of the Workshop "Heavy Ion Physics at the AGS" (HIPAGS '96), Wayne State University 1996, WSU-NP-96-16.

5. P. Braun-Munzinger, J. Stachel, J.P. Wessels, and N. Xu: Phys. Lett. B344 (1995) 43; Phys. Lett. B365 (1996) 1.

6. P. Braun-Munzinger, J. Stachel: Nucl. Phys. A606 (1996) 320.

7. H. Bebie, P. Gerber, J.L. Goity, and H. Leutwyler: Nucl. Phys. B378 (1992) 95.

8. H. Sorge: SUNY-NTG 97-1, nucl-th/9701012, Phys. Lett. B (1997) in print.

9. H. Sorge: Z. f. Phys. C67 (1995) 479.

10. H. Sorge, M. Berenguer, H. Stöcker, and W. Greiner: Phys. Lett. B289 (1992) 6.

11. N. Armesto, M.A. Braun, E.G. Ferreiro, and C. Pajares: Phys. Lett. B344 (1995) 301.

12. C. Bormann (for the NA49 Collab.): Contrib. to the Proceedings 'Strangeness In Quark Matter 1997', Santorini (Crete) April 1997.

13. H. Sorge: Phys. Rev. C52 (1995) 3291.

14. A. Capella: Phys. Lett. B364 (1995) 175.

15. K. Werner: Phys. Rep. 232 (1993) 87.

16. K. Geiger, D.K. Srivastava: nucl-th/9706002, K. Geiger, R. Longacre: nuclth/9705041. 
17. T.S. Biro, P. Levai, and J. Zimanyi: Phys. Lett. B347 (1995) 6.

18. J. Rafelski, J. Letessier, and A. Tounsi: Phys. Lett. B390 (1997) 363.

19. A. Jahns et al.: Phys. Rev. Lett. 72 (1994) 3464.

20. S.H. Kahana, Y. Pang, T. Schlagel, and C.B. Dover: Phys. Rev. C47 (1993) R1356.

21. H. Sorge: Phys. Lett. B344 (1995) 35.

22. J. Cleymans: Talk presented at the 'Third International Conference on Physics and Astrophysics of Quark-Gluon Plasma', March 1997, Jaipur, India, nucl-th/9704046.

23. L.V. Bravina, I.N. Mishustin, N.S. Amelin, J.P. Bondorf, and L.P. Csernai: Phys. Lett. B354 (1995) 196.

24. V. Topor Pop et al.: Phys. Rev. C52 (1995) 1618; M. Gyulassy, V. Topor Pop, and X.N. Wang: Phys. Rev. C54 (1996) 1497.

25. M. Gazdzicki, U. Heinz: Phys. Rev. C54 (1996) 1486.

26. H. Sorge, L. Winckelmann, H. Stöcker, and W. Greiner: Z. f. Phys. C59 (1993) 85.

27. S. Abatzis et al. (WA85 Collab.): Phys. Lett. B2400 (1997) 239.

28. G. Odyniec (for the NA49 Collab.): Contrib. to the Proceedings 'Strangeness In Quark Matter 1997', Santorini (Crete) April 1997.

29. Courtesy of C. Ogilvie (see his contribution to the proceedings) and J. Dunlop who made the comparison with RQMD.

30. J. Letessier, A. Tounsi, U. Heinz, J. Sollfrank, and J. Rafelski: Phys. Rev. D51 (1995) 3408. M. Tazdzicki (for the NA35 Collab.): Nucl. Phys. A590 (1995) 197c.

31. N. Davidson, H. Miller, R. Quick, and J. Cleymans: Phys. Lett. B255 (1991) 105.

32. H. Sorge: Phys. Lett. B 373 (1996) 16.

33. H. Sorge, R. Mattiello, H. Stöcker, and W. Greiner: Phys. Lett. B271 (1991) 37.

34. See V. Metag's contribution.

35. L.Ya. Glozman, D.O. Riska: Phys. Rep. 268 (1996) 263.

36. G.Q. Li, C.-H. Lee, and G.E. Brown: nucl-th/9706057.

37. J. Sollfrank, P. Huovinen, M. Kataja, P.V. Ruuskanen, M. Prakash, and R. Venugopalan: Phys. Rev. C55 (1997) 392.

38. A. Leonidov, M. Nardi, and H. Satz: Nucl. Phys. A610 (1996) 124c.

39. E. Shuryak: hep-ph/9704449.

40. G.Q. Li, C.M. Ko, and B.A. Li: Phys. Rev. Lett. 74 (1995) 74. 